\title{
Diabetic Retinopathy Screening In Human Eyes Using Image Processing and Segmentation
}

\author{
Ashima Gambhir, Deepthi Sehrawat, Harsh Kumar, Yashvir Singh, Hemant Saini,
}

\begin{abstract}
Diabetic Retinopathy is a complication in which the retina of the eye gets damaged due to secretion of fluid from blood vessels to retina, It may also cause vision loss. It occurs due to damage of blood vessels in the retina. In this approach, we suggest an ANN method to detect the presence of unnatural new blood vessels. This new concept is used for distinguishing age-related problems i.e., agerelated macular degeneration (AMD). Our new improved matched filters distinguishes the higher efficiency in true up positive feedback and less efficiency in false observation in previously used filter based on vessel separation. So the aim is to provide an automated, easy to use, UI based detection system preparing the raw images to be processed by applying general operations such as cropping and resizing the images and applying noise reduction filters, finding global mean and using it for thresholding and also applying a pad for distinct borders to avoid confusion. The DR can be observed at rapid levels simply and destruction of the retina will be less. The key research methodology is stated that describing patterns that report the characteristics of an entire image such as outcome arranged images are graceful with dissimilar filters, geomorphologic tools, noise contraction, background separate and vessel separation. Comparison of ANN technique, and support vector machine (SVM) technique used for the classification and grading technique based on supervised learning.
\end{abstract}

KEYWORDS- Diabetic Retinopathy, DR, SVM, ANN, classification, AMD (Age-related macular degeneration).

\section{Manuscript received April 27, 2020}

Ashima Gambhir, Assistant Professor,Department of Computer Science,Amity University Haryana, India (email: agambhir@ggn.amity.edu)

Deepthi Sehrawat, Assistant Professor,Department of Computer Science,Amity University, Haryana, India,

Harsh Kumar, Student,Department of Computer Science, Amity University, Gurugram, Haryana, India,

Yashvir Singh, Student,Department of Computer Science, Amity University, Gurugram, Haryana, India,

Hemant Saini, Student, Department of Computer Science, Amity University, Gurugram, Haryana, India,

\section{INTRODUCTION}

Diabetic retinopathy is a medical complication that affects eyes and is caused when a patient is diabetic for a long period of time[6]. A substantial amount of people affected with diabetes over a period of 10 years or more are at risk to develop at least some of the symptoms, if not all, of diabetic retinopathy. his also proves to contribute to around 5\% of total cases of blindness, especially in the developing countries due to lack of proper treatment. Diabetic retinopathy can prove to be fatal to retina and optic nerves. If this condition is not found and treated in premature stage then, it can lead to formation of exudates, microaneurysm, which in fact are considered to be the primary indicators of diabetic retinopathy and penetration of blood vessels into vitreous humor and haemorrhage which can ultimately lead to partial or absolute blindness. Early diagnosis can avert most of these symptoms using screening periodically. Despite the conduction of the screening process and supervision under pristine eyes of the professional ophthalmologist, diabetic retinopathy can go undetected easily because of lengthy and tedious processes and chances of delayed results. Diagnosticians have classified the condition into two broad categories i.e. Proliferative and Non-Proliferative, which can further be classified into various classes. And also some age related complications in the eyes may be misdiagnosed as diabetic retinopathy. Our aim for this paper is to present automated, easy to use, UI based detection system preparing the raw images to be processed by applying general operations such as cropping and resizing the images and applying noise reduction filters, finding global mean and using it for thresholding also applying padding for distinct borders to avoid confusion. Furthermore the preprocessed images are further subjected to filtering and processing with different filters, morphological tools, noise reduction, background removal, masking, vessel extraction and much more, which is described in detail later on. Then finally the images are used for classification, and as there are numerous classification and grading techniques based on supervised learning we are going to use the two most commonly used techniques i.e. ANN (artificial neural network) and SVM (support vector machine) to compare their accuracy on different set parameters which are accuracy, sensitivity and specificity on retinal images acquired by screening to detect diabetic retinopathy in early stages to grade and classify and distinguish age related problems and Diabetic retinopathy to help decrease the damage caused to the patient, as a study suggests that $90 \%$ of the new cases of DR can be slashed by providing proper care and treatment, whereas regular checkup is imperative. The dataset used is a combination of both Indian and international repository of the retinal fundus images for training and testing. The extensive and 


\section{Diabetic Retinopathy Screening In Human Eyes Using Image Processing and Segmentation}

mixture of these two will provide us with a holistic view of the approach.

\section{LITERATURE REVIEW}

In paper [1], the authors publish their paper in the year of 2015 and their name is Deepthi k Prasad, Vibha and Venuggopal K R and paper name is Early Detection of Diabetic Retinopathy from digital retinal fundus images. They used ANN and MATlab technology in their paper and they get the data set from DIARETDB1 only 89 images. The Paper [2] was published on 2016 and their author name is Darshit Doshi, Aniket Shenoy and Deep Sidhpura and paper name is Diabetic Retinopathy Detection using Deep Convolutional Neural Networks and they used CNN and Image Magick and the Python library OpenCV technology and they had the dataset of 35,126 images and they get the data set from EyePACs and in the paper [3], the author publish their paper in the year of 2017 and author names is S.D.Shirbahadurkar ,V. M. Mane and D. V. Jadhav and paper name is A Modern Approach to Detection of Diabetic Retinopathy and Teleophthalmology technology used by them and they get the dataset from DIARETDB1 (89 images) In the paper[4], the authors publish their paper in the year of 2018 and the author names is Avula Benzamin ,Chandan and Chakrabort and their paper name is Detection of Retinal Fundus Images using Deep Learning and they used Tensorflow deep learning framework technology and they had dataset of 89 images (DIARETDB1) and in the paper[5] the authors publish their paper in the year of 2019 and the author names is Xianglong Zeng, Haiquan Chen, Yuan Luo \& Wenbin Ye and their paper name is Automated Diabetic Retinopathy Detection Based on Binocular Siamese and they used CNN to detect RDR with the help of deep learning and they get the dataset of 35126 images (KAGGLE).

\section{METHODOLOGY}

Before performing any operations or processing on the images, they are first needed to be converted into a generalized global format. As the fundus images in the data set have large inconsistencies, such as variable brightness, contrast, resolution and colour spectrum[1], since most of them belong to different databases and repositories and are procured with different equipment in different environments. Therefore, to convert the fundus images into generalized form, they undergo several preprocessing operations and filtering.

\section{A. Image Preparation}

The steps followed to prepare the fundus images for the processing and classification of the disease are depicted in the flowchart, ranging from resizing, noise removal, and padding to masking.



Fig 1: Flowchart of preparation of images

i. Image Resizing:The fundus images are resized because of the variable resolution of these images and mainly for the sole purpose of faster computation. The images are resized into $200 \times 200$ pixels.

ii. Noise Reduction \& Arithmetic Operations: Resizing images might induce some noise, so to remove any unwanted noise 'Laplacian' filter is used and also the image is converted into a matrix of long integer type to calculate global mean of the image.

iii. Threshold \& Relative Mean: The global mean is used to filter objects below the mean value and also objects smaller than 2 pixels which will eliminate smaller insignificant objects.

iv. Image Padding \& Masking: Padding and masking of the images is necessary, they help in removing the background and isolate the image and acts as a border for filtering and also to create space between two shown images.

The images obtained by applying these instructions are finally ready for the processing and for segmentation.

\section{B. Image Processing}

The resulting images obtained from the above mentioned process is not in the terminal state, furthermore processing is required. The process is shown in the following flowchart. 


\section{Pre-processinglmages}
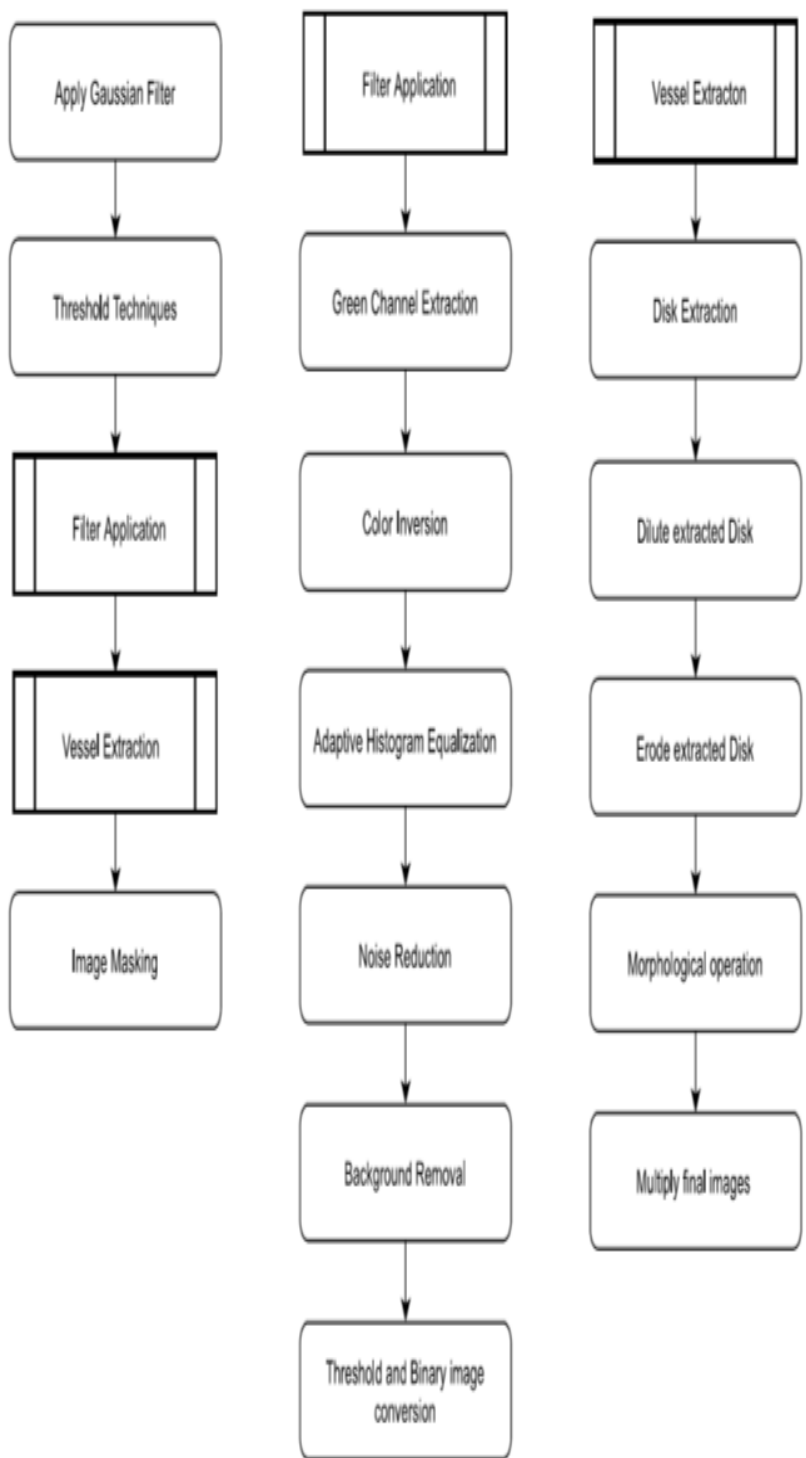

Fig 2: Flowchart of pre-processing of images

i. Thresholding \& Noise Removal: For noise removal we've used the 'Gaussian' filter and 'graythresh' function for threshold.

ii. Filter Application: Here the green channel is extracted from the images because in the green channel the blood vessels are manifested clearly. Color inversion and Adaptive Histogram Equalization (AHE) techniques are used to highlight vessels.

iii. Background Removal \& Noise Reduction: Again noise removal filters such as gaussian and laplacian are applied to remove noise. The background of the image is to be removed to extract easily visible blood vessels.

iv. Threshold and Binary image conversion: The successive thresholding of the image provides us better distinction and contrast in the image.
The resultant image is converted from grayscale to black and white or binary image.

v. Disk extraction \& removal: Here 'Disk' implies to the optic disk which covers a significant amount of area and may also act as an outlier in the Classification stage. So, the disk is extracted and removed using morphological tools such as 'dilute' and 'erode'.

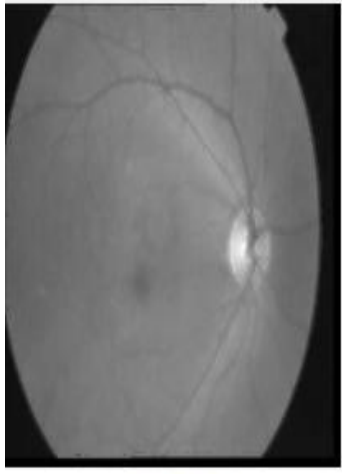

\section{Grey-Scale Image Extracted Disk}

vi. Multiply final images: Multiplying the images using the matrix will overlap the extracted vessels and the original unprocessed image to provide contrast and help users to observe the results with their own eyes.

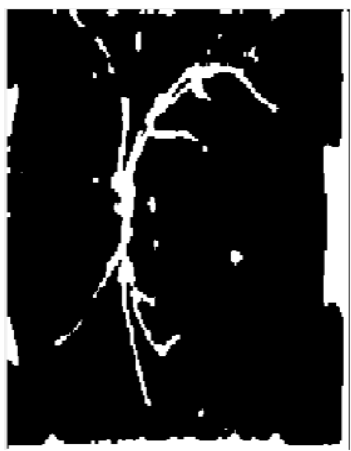

Extracted Vessel

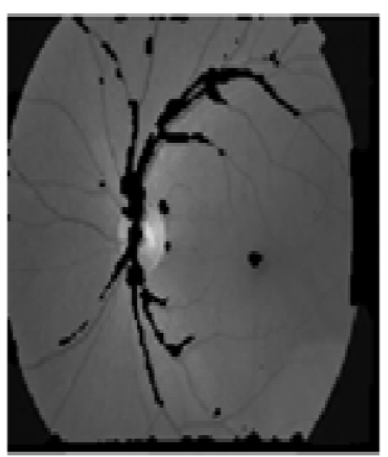

Multiplied Image
Fig 4: Extracted vessels and Multiplied Image

\section{Training \& Testing Image Classification}

For our classification model, we used two different models being Support Vector Machine(SVM) and Neural Network and compared them head to head against each other to check their efficiency on three major factors i.e Accuracy, Sensitivity and Specificity.

The dataset was taken from Kaggle containing fundus images from IRDIA of indian subjects and from IEEE dataport repository for additional data. For SVM models a datatrain of 50 images is used for training. Correlation function used in these models is the 'Gray-level cooccurrence matrix'(GLCM) and is based on the 'Energy', 


\section{Diabetic Retinopathy Screening In Human Eyes Using Image Processing and Segmentation}

'Homogeneity' feature. The comparison of the two is based on 4 functions that are False-positive, False-negative, Truepositive, True-negative.

\section{RESULT \& DISCUSSION}

The results of the comparison of the models are then plotted on the chart with respective factors along with the classification type, i.e type 1 (Normal), type 2(DR), type 3 (AMD-age related macular degeneration), original image, pre-processed image, processed image, extracted vessel and Binary image of the data. Following are the demo results of the test image for representation purpose, these different graphs portray accuracy, sensitivity and specificity of a single test image.

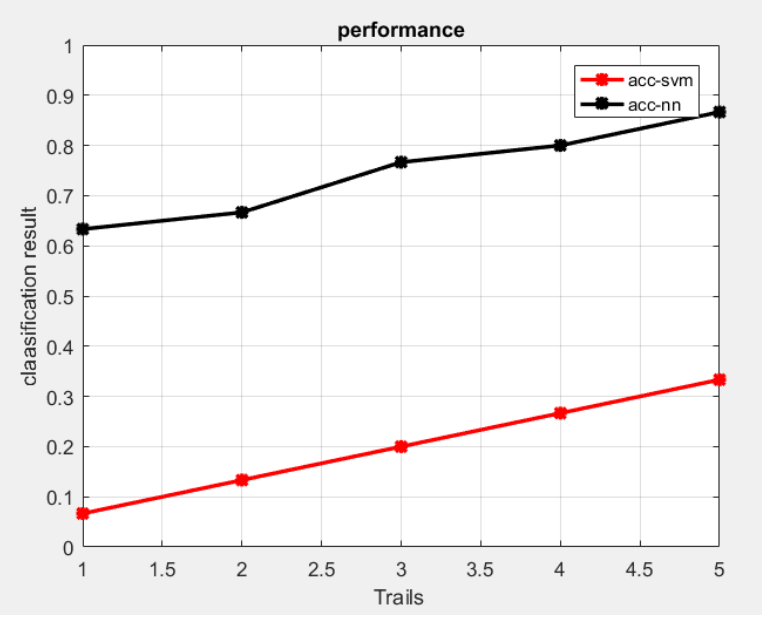

Fig 5: Performance graph of accuracy

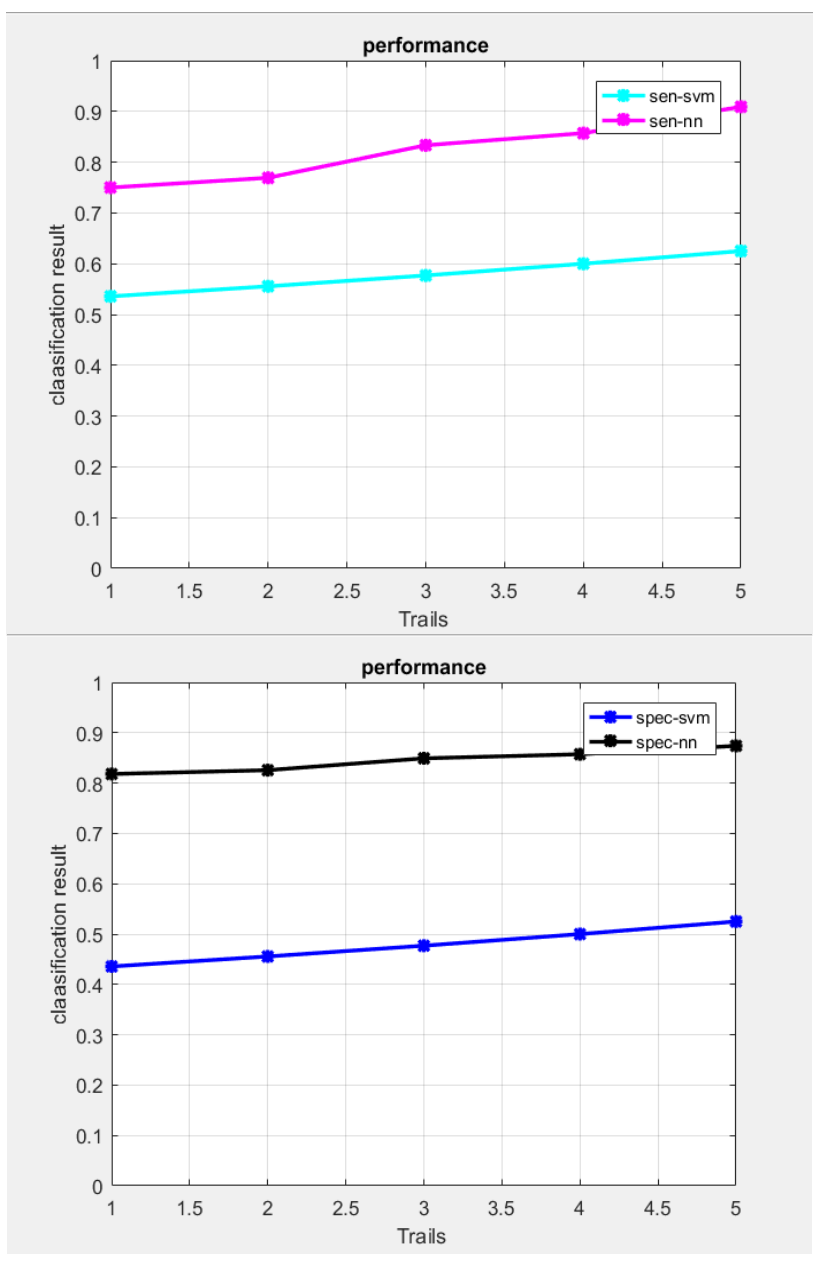

Fig 6: Performance graph of specificity \& Sensitivity

\section{CONCLUSION}

In this project, a neural network model compared with SVM model is used for automatically recognize the DiabeticRetinopathy by using image processing and it also gives the different review as per their different phases and it also helps to detect the DR disease in early stages.

In this proposed project, the system compares the patient image with the normal image and after testing (comparing all the cases) we get the output as per their classes as and it also provides the performance graphs as (Sensitivity, Specificity and Accuracy) and we also added a new concept of AMD (Age-related macular degeneration) in our project. The evaluation result of the database has proved to be competent enough with an accuracy of $86.5 \%$ and with the sensitivity of $90 \%$ and with the specificity of $87.5 \%$. It is a fact that with better and accurate diagnosis, it will be more helpful for the doctors for better treatment. So diagnostic should aim towards accuracy for an effective treatment regimen. In this project we were able to set up better accuracy in our results. The proposed system can be improved in the future by using better classification techniques, extensive dataset and also higher processing power to process images in higher pixel amounts.

\section{REFERENCES}

[1] Deepthi K Prasad , Vibha , Venugopal K R, Early Detection of Diabetic Retinopathy from Digital Retinal Fundus Images, 2015

[2] Darshit Doshi ,Aniket Shenoy ,Deep Sidhpura Diabetic Retinopathy Detection using Deep Convolutional Neural Networks, 2016

[3] S.D. Shirbahadurkar, V. M. Mane , D. V. Jadhav. A Modern, 2017

[4] Approach to Detection of Diabetic Retinopathy. Avula Benzamin , Chandan Chakraborty. Detection of Retinal Fundus Images using Deep Learning,2018

[5] Xianglong Zeng, Haiquan Chen, Yuan Luo \& Wenbin Ye. Automated Diabetic Retinopathy Detection Based on Binocular Siamese, 2019

[6] S. Choudhury, S. Bandyopadhyay, S. K. Latib, D. K. Kole, C. Giri. "Fuzzy C means based feature extraction and classification of diabetic retinopathy using support vector machines", 2016 International Conference on Communication and Signal Processing (ICCSP), 2016

[7] R. Casanova, S. Saldana, E. Y. Chew, R. P. Danis, C. M. Greven, and W. T. Ambrosius, "Application of random forests methods to diabetic retinopathy classification analyses," Plos One, vol. 9, no. 6, p. e98587, 2014.

[8] Kaggle, "Diabetic retinopathy detection," https://www.kaggle.com/c/diabetic-retinopathydetection/, July 27, 2015, accessed May 7, 2018.

[9] V. Gulshan, L. Peng, M. Coram, M. C. Stumpe, D. Wu, A. Narayanaswamy, S. Venugopalan, K. Widner, T. Madams, and J. Cuadros, "Development and validation of a deep learning algorithm for detection of diabetic retinopathy in retinal fundus photographs." Jama, vol. 316, no. 22, p. 2402, 2016.

[10] N. Srivastava, G. Hinton, A. Krizhevsky, I. Sutskever, and R. Salakhutdinov, The Journal of Machine Learning Research, vol. 15, no. 1, pp. 1929-1958, 2014.

[11] S. Chopra, R. Hadsell, and Y. LeCun, "Learning a similarity metric discriminatively, with application to 
face verification," in Computer Vision and Pattern Recognition, vol. 1, pp. 539-546,2015

[12] S. R. Flaxman, R. R. Bourne, S. Resnikoff, P. Ackland, T. Braithwaite, M. V. Cicinelli, A. Das, J. B. Jonas, J. Keeffe, J. H. Kempen et al., "Global causes of blindness and distance vision impairment 1990-2020: a systematic review and meta-analysis," The Lancet Global Health, vol. 5, no. 12, pp. e1221-e1234, 2017.

[13] G. Quellec, K. Charria"Re, Y. Boudi, B. Cochener, and M. Lamard, "Deep image mining for diabetic retinopathy screening," Medical Image Analysis, vol. 39, pp. 178-193, 2017. 